\title{
Metacognitive Development and Conceptual Change in Children
}

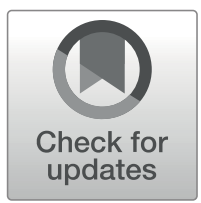

\author{
Joulia Smortchkova $^{1} \cdot$ Nicholas Shea $^{2}$
}

Published online: 22 April 2020

(C) The Author(s) 2020

\begin{abstract}
There has been little investigation to date of the way metacognition is involved in conceptual change. It has been recognised that analytic metacognition is important to the way older children (c. 8-12 years) acquire more sophisticated scientific and mathematical concepts at school. But there has been barely any examination of the role of metacognition in earlier stages of concept acquisition, at the ages that have been the major focus of the developmental psychology of concepts. The growing evidence that even young children have a capacity for procedural metacognition raises the question of whether and how these abilities are involved in conceptual development. More specifically, are there developmental changes in metacognitive abilities that have a wholescale effect on the way children acquire new concepts and replace existing concepts? We show that there is already evidence of at least one plausible example of such a link and argue that these connections deserve to be investigated systematically.
\end{abstract}

\section{Introduction}

It is often thought that various kinds of metacognition are central to the way children learn, especially in an educational setting (Blank 2000; Thomas 2012). Increasing metacognitive sophistication supports their learning processes. It is part of what makes their thinking become more mature. In this paper we explore whether metacognition supports a specific kind of developmental process - the process that changes the child's conceptual resources. In particular, we ask whether changes in metacognitive abilities lead to changes in the way the child's concepts alter during learning and development.

What is conceptual change? Take the case of how children's concept of the earth is revised during the school years. The majority of first-graders think of the earth as a flat

Joulia Smortchkova

joulia.smortchkova@philosophy.ox.ac.uk

1 Faculty of Philosophy, University of Oxford, Woodstock Road, Oxford, UK

2 Institute of Philosophy, School of Advanced Study, University of London, Senate House, Malet

Street, London WC1E 7HU, UK 
disk at the centre of the solar system (Özsoy 2017). During the education process children revise their concept of earth until they think of it as a sphere rotating around the sun (Vosniadou and Brewer 1992). This outcome is a difficult achievement. The path to change is full of cognitive obstacles: some of them come from innate cognitive constraints (Carey 2009), some from everyday experiences and observations that inform children's framework theories (Wellman and Gelman 1998), some others from individual variations in learning, and from motivational factors. As a result, changes in children's concept of earth do not occur effortlessly, but are the outcome of a learning process. That process involves, among other factors, explicit reflection on the beliefs associated with the original concept and on the way new pieces of information fail to integrate within the framework of the original concept (Vosniadou and Brewer 1992). Explicit reflection is a metacognitive process.

While in the earth example we can identify a (relatively) clear case of conceptual change, what exactly counts as a conceptual change depends one's theory of concepts and of conceptual change. In this paper we will consider cases of conceptual change that are simpler than this example. We will also consider cases of metacognition that are less reflective than the explicit and rich metacognitive process involved in changing one's concept of earth - in particular, we will explore the possible role of procedural metacognition in conceptual change, such as a feeling of confidence that is attached to the old concept of earth and that might slow down the process of change. After briefly sketching the notions of conceptual change and metacognition we will work with, we define them in greater detail in sections 2 and 3, respectively.

Metacognition can be defined via its functional role in cognition (Proust 2010) as the set of processes that monitor and control cognitive activity (which includes perceiving, planning, categorising, remembering, learning, desiring, reasoning, intending and acting). Monitoring refers to the processes involved in the supervision of cognitive activities while they unfold, and in evaluating their progress and success in respect to the goal of a specific task; control refers to the top-down management and regulation of cognitive processes that makes use of feedback from monitoring, of the goals of the person, and of her background beliefs and theories about the cognitive process in question and her own abilities (Koriat 2016; Proust 2010).

Metacognition plays a role in conceptual thought. Concepts are mental representations that are involved in a variety of cognitive processes, such as categorising, recalling from semantic memory, and making inferences. There are at least three ways in which metacognition is involved with conceptual thought: metacognition attached to concept use (Shea 2019), metacognition attached to the bodies of information associated with a concept ${ }^{1}$; and metacognition involved in the process of conceptual change. Here we consider only the last of these.

Conceptual change is an area of cognitive development that has been much-studied by developmental psychologists (Carey 2009; Carey and Spelke 1994; Keil 1989; Opfer and Siegler 2004). This paper asks about the role of metacognition in the process of conceptual change, including in concept acquisition, concept revision, and concept replacement. So far there has been little systematic investigation of the way metacognition is involved in conceptual change. Here we make the case that there are likely to be interesting connections between metacognitive development and conceptual

\footnotetext{
${ }^{1}$ E.g. to prototypes, exemplars, theories, semantic memories and sensorimotor expectations.
} 
development. It is plausible that changes in the child's metacognitive abilities can have an across-the-board effect on the way they acquire new concepts and change their preexisting concepts. A metacognitive change may usher in a new stage of conceptual development.

That is an enticing prospect. There is currently relatively little direct evidence unsurprisingly, since it has not been sought. Here we suggest there is preliminary evidence for one previously-unrecognized connection between metacognitive development and conceptual change, and we motivate the search for more.

The paper is structured as follows. In section 2 we discuss types of conceptual change and the theory of concepts we're working with. In section 3, we set out the important distinction between two different varieties of metacognition: analytic and procedural. In section 4, we overview how metacognition develops across the ages with the aim of identifying metacognitive developments that could play a role in the process of conceptual change. In section 5, we develop a hypothesis about the relation between metacognitive overconfidence and resistance to change. In section 6 , we discuss the relation between the acquisition of a theory of mind and metacognitive development. In section 7 we discuss the role of analytic metacognition in thinking with analogies as part of the process of conceptual change. In section 8 we formulate some predictions. Finally, section 9 is a brief conclusion.

\section{Conceptual Change}

How radical does a change of a concept have to be to count as an interesting conceptual change? For our purposes we don't need to presuppose that there is any hard-and-fast answer to that question. We take our lead from the kinds of conceptual change that have been of interest in developmental psychology, for example: moving from a flat to a spherical conception of the earth (Vosniadou 1994), distinguishing between weight and density (Smith et al. 1992), and distinguishing between properties of objects and of stuffs (Carey and Spelke 1994; Spelke and Van de Walle 1993). While there are interesting parallelisms between conceptual change at the individual level in development and at the group level in history of science (Carey 2009; Thagard 2018), we restrict our attention to changes at the individual level.

Asking how an individual's concepts change raises the broader question of how concepts should be individuated. Developmental psychologists working on conceptual change usually appeal to the theory theory of concepts (Carey 2009): concepts are bodies of beliefs and knowledge inter-connected in a web of theoretical postulates and observations, modelled on scientific theories. Conceptual change is thus a process of change in theoretical understanding. We want to discuss conceptual change in a way that is not committed to the theory theory of concepts. The reason is that conceptual change could occur not only for concepts as theories, but also for concepts as prototypes or exemplars, for concepts as definitions (if there are any), or for concepts as atomic symbols with associated bodies of information. We will be working with a more inclusive approach to concepts, where by concept we mean a mental representation that encodes information about a given category, including information that governs how it is applied to the world and used in inference. This information may consist of beliefs, inferential dispositions and/or sensorimotor expectations. Thus, our 
discussion of conceptual change will not be hostage to the truth of a particular theory of concepts. Our task is not to solve the controversy of what counts as a change in a concept rather than as a change of concepts.

The examples of conceptual change at the start of this section all involve changing some kind of theory or schema, rather than just changing a single belief. One way of talking about conceptual change, without having to resolve the vexed question of how concepts should be individuated, is to focus on coarse- rather than fine-grained individuation of cognitive structures (Strevens 2019, p. 64). Psychologists working on conceptual change often focus on representational schemes rather than on a particular set of representations (such as a description, a theory, a prototype or an exemplar). Conceptual change occurs when the scheme corresponding to a set of terms (e.g. heat, living being, natural number terms) changes in a major way (Strevens 2019, p. 65). We can talk about these cases by saying what has changed in each case without needing a general way of individuating conceptual schemas, and without being committed to a particular way of individuating concepts. Following Thagard (2018), we will take conceptual changes to include: addition of new information to a concept, deletion of information from a concept, simple reorganisation (differentiation, coalition, decomposition), revisionary reorganisation, hierarchy redefinition, and also changes in the domain of application of a concept (Clement 2013). Conceptual change from this broad perspective includes both changes in the information encoded with a concept, and more radical changes such as replacement of a concept or acquisition of a second concept.

Our catholic approach to theories of concepts is close to the spirit of current pluralistic approaches to concepts (Weiskopf 2009). Recent theories of concepts conceive them either as complex hybrids which include different bodies of information (Vicente and Martínez Manrique 2014) or as plural categories that contain as subcategories different bodies of information (Weiskopf 2009). The body of information used depends on the task and on the context at hand. ${ }^{2}$ For example, a concept may be applied on the basis of surface features (e.g. for quick visual categorisation) or on the basis of inferences about an underlying essence (e.g., for making inferences about members that fall under the concept) (Keil 1989). A change from the first psychological structure to the second counts as a conceptual change, and does so irrespective of whether it should count as a change in a concept or a change of one concept into another. Similarly, acquiring concepts of natural numbers is a matter of acquiring a new psychological structure: an unbounded collection of concepts about numerosity that are inferentially linked via the successor relation (Carey 2009).

We have listed types of conceptual change. But what are the mechanisms of conceptual change? The developmental literature offers some proposals for the mechanisms. One possible mechanism is maturation, where innate learning constraints express themselves in the right environment (Carey and Spelke 1994). Another theory is canalization: first a concept is acquired and then children learn about the things that the concept refers to from experience (Carey 2009). There are also proposals intermediate between these two, such as Xu and Tenenbaum's Bayesian learning approach that

\footnotetext{
$\overline{2}$ This is compatible with specific bodies of information involving metacognition differently: theories might involve analytic and inferential forms of metacognition, while prototypes might involve procedural forms of metacognition, for example the principle of the least cognitive effort. One should distinguish between metacognition associated with the body of information, and metacognitive processes involved in conceptual change. Our focus here is on the latter. We are grateful to Joëlle Proust for helping us clarify this issue.
} 
integrates learning constraints and learning from experience (Xu and Tenenbaum 2007). Finally, some types of conceptual change are supported by explicit instruction in an educational setting. The first three mechanisms involve learning only at the object level, while the last kind is widely taken to involve metacognition (of an explicit, analytic kind). Here we are interested in whether metacognition (of some kind) also plays a role in other kinds of mechanisms of concept-acquisition and concept-change.

\section{Procedural as Well as Analytic Metacognition}

The idea that developmental changes in metacognition drive changes in cognitive performance in general has long been a mainstay of educational psychology (Flavell 1979). As well as memory and mnemonic strategies, this work has focused on aspects of the school curriculum like reading, language learning, and science (Haukås et al. 2018; Thomas 2012; Van Kraayenoord 2010). For example, work on science education has found that understanding one's own mental states and theories is crucial to the way children learn new scientific knowledge, as is the reflective use of analogies to provide new ways of thinking about a domain (Carey 2000; Clement 2013; Hennessey et al. 1996; Thagard 1992). Understanding one's own mental states comes with the acquisition of a theory of mind at around 4 years of age (Ruffman and Perner 2005). While this is an important developmental stage, it is unlikely that it plays an across the board role in conceptual change (for reasons outlined in section 6).

Relatively little work on metacognition in educational settings has focused specifically on conceptual change, despite the wealth of developmental psychology on the topic, much at earlier ages (for exceptions see (Sinatra and Pintrich 2003; Vosniadou 2007)). Nonetheless, our examination of existing research on the two topics suggests that they are likely to be connected in illuminating ways. This involves looking at whether metacognition is involved in early concept acquisition, in pre-school children and children at the beginning of school, as well as for concepts acquired at school age like concepts of rational numbers and biological categories. We need to look carefully at metacognition in younger children, before the age (c. 8-12 years) when they have explicit knowledge about their memory capabilities and the use of explicit analogies. Explicit knowledge about memory capabilities and reflective use of analogies are rather late achievements (Schneider and Pressley 2013), but children change their concepts even before that age.

The other way we need to enlarge our focus is to examine procedural and not only analytic metacognition (Flavell and Wellman 1975; Koriat 2016; Proust 2013). Analytic metacognition encompasses explicit beliefs about first-order mental states and processes, for example about effective memory strategies (Son 2004). Analytic metacognition draws on people's folk beliefs about how the mind works, which may be false. Procedural metacognition is based on feelings attached to cognitive processes, like a feeling of fluency during recall or a feeling of knowing when considering a question (these feelings can be collected under the broad notion of 'noetic feelings', see (Proust 2013)). Unlike analytic metacognition, procedural metacognition is thought to exist in non-human animals (Beran et al. 2012; Hampton 2009). Feelings can be used directly to guide behaviour, or they can be used to formulate judgements, for example a feeling of fluency can be the basis for a judgement of confidence (Schwarz 2012). 
What is the connection between procedural metacognition and conceptual change in the early stages of development? Some forms of procedural metacognition develop early (Goupil and Kouider 2016; Goupil et al. 2016). This opens up the possibility that procedural metacognition might be involved in early stages of conceptual development, whether it is acquisition, formation, or replacement of concepts. Changes in procedural metacognition, and not only the development of analytic metacognition, may open up across-the-board changes in the way concepts are learnt. Yet, procedural metacognition has been largely overlooked in the educational literature on learning, even if it is now recognised that procedural metacognition might be effective in guiding behaviour even in the absence of explicit metacognitive reports (Kim et al. 2016).

One form of procedural metacognition that is relevant here is confidence (estimation of the correctness of one's knowledge). Pre-school children exhibit generalised overconfidence, even in the face of disconfirming evidence (Schneider 1998). Once a child has acquired a functioning concept in a domain (e.g. LIVING THING), generalised overconfidence could present an obstacle to changing it when new evidence is provided, because the overconfident child sees no reason to revise her concept. ${ }^{3}$

This evidence suggests the possibility that a metacognitive change such that the child is better able to recognise inconsistencies and reduce their confidence as a result may be an important gateway to conceptual change: to gaining a new concept to supplement or replace the defective one, for example when children revise their concept of earth from being flat to being round. That is one case where a developmental change in metacognition plausibly leads to wholescale changes in what kinds of concept are acquired and how they are learnt. Our hypothesis in section 5 will be that lifting the confidence bias as a functional connection between (procedural) metacognition and conceptual change, and that one way of reducing the confidence bias is via the detection of inconsistencies. That is just one possible connection, but it shows that it will be worthwhile systematically to investigate this and other potential connections between metacognitive changes and stages of conceptual development. In the next section we review the literature on metacognitive development in general, with the aim of identifying metacognitive changes that are candidates to have an impact on conceptual development.

\section{Metacognitive Development}

The two types of metacognition identified in the previous section are likely to relate to conceptual change in different ways: while analytic metacognition is important later in development, procedural metacognition may play a role for conceptual change much earlier. This is mirrored by their dissimilar maturation paths: analytic and procedural metacognition show different developmental patterns (Schneider and Löffler 2016).

\footnotetext{
${ }^{3}$ We need to distinguish between self-confidence and confidence. Self-confidence refers to a person's trust in her own ability to succeed and learn across different tasks. In a learning environment, self-overconfidence might play an adaptive role in fostering motivation, in stimulating a child (or an adult) to learn and explore more (Chen and Schildberg-Hörisch 2019). Confidence occurs in the context of a specific task, by measuring the person's estimation of the correctness of her response. An over-confident person (in the context of a task) would think she has given a correct response when she has made a mistake. Self-overconfidence and overconfidence can potentially be studied independently.
} 
Analytic metacognition, initially poor, increases substantially during school-age years, often as a result of teaching. Procedural metacognition that monitors cognitive processes, for example the feeling of confidence, stretches back to early childhood (Balcomb and Gerken 2008).

This section looks at the way metacognition in general develops in childhood. Not all of these changes will be relevant to conceptual development, but our aim is to identify potential candidates: metacognitive changes that may have a large-scale effect on the way concepts are acquired or changed in development. Metacognition of memory, which has been studied extensively, offers a useful literature; and we look first at children's analytic metacognition about memory. When starting school, children overestimate their own memory capabilities and show little appreciation of how memory varies (Schneider and Löffler 2016). By 9 to 11 years old, children know that memorisation skills vary from person to person and from situation to situation (Kreutzer et al. 1975). Knowledge of memory strategies also improves in the school years. It is not until 8-, 9- or 10-years-old that children appreciate that taxonomic or semantic organisational structure (for example, that items such as "chair-table" belong to the semantic category "furniture") confers a memory advantage (Moynahan 1978; Sodian and Schneider 1999). Knowledge of the combined influence of variables like effort and the number of items to be memorised continues to develop through the school years into adolescence (Schneider and Pressley 2013).

Outside the domain of memory, other forms of analytic metacognition develop during the schooling years. One such form is the use of explicit analogies. Explicit analogising involves reflecting on one's way of thinking about one domain and selfconsciously deploying it to think about a different domain. This is one form of metacognition that has been thought to play a role in conceptual development, typically in the later stages of elementary school (8-12 years old), for example in acquiring a mature conception of the circulation system (Mason 1994), of rational numbers (Carey 2009, ch. 9), and of weight and density (Carey 2009, ch. 10).

In short, analytic metacognition does develop in the school age years, and is used self-reflectively in learning subtler scientific and mathematical concepts in the later years of primary school. What about changes in procedural metacognition?

Procedural metacognition is observed in infants in the form of confidence or the monitoring of uncertainty. 20-month-old infants given the chance to ask for help in a memory task do so strategically, making requests selectively to avoid making errors (Goupil et al. 2016). 12- and 18-month-old infants exhibit a neural signature that they evaluate their decision in a memory task, and use their uncertainty adaptively to change their mind when given the opportunity (Goupil and Kouider 2016). When their confidence is low they also search less long for a hidden object. These experiments go beyond the widespread finding that infants are sensitive to violation-of-expectation, which happens when an unexpected event occurs, without the need for a metacognitive evaluation (Aslin 2007). They show that even one-year-old infants monitor their certainty and uncertainty in a decision - a form of procedural metacognition. By two years old children are monitoring their own state of knowledge or ignorance, communicating it verbally and non-verbally, and using this monitoring as a basis for requesting more information (Geurten and Bastin 2019; Harris et al. 2017). Slightly older children can be directly asked to rate their confidence in an answer they have given to memory question. Even 3-year-olds show some metacognitive sensitivity, giving higher 
confidence ratings for correct than for incorrect answers (Balcomb and Gerken 2008; Lyons and Ghetti 2011). ${ }^{4}$

Metacognitive feelings can be used to formulate judgements of confidence. There is a developmental trend for judgments based on procedural metacognitive experiences and feelings. The accuracy of trial-by-trial confidence judgements is initially low. It improves considerably in the early school years so that by 7 or 8 years old children can accurately report whether an answer is likely to be correct (Krebs and Roebers 2012; Von der Linden and Roebers 2006), provided the questions are not asked in a misleading way; although children, like (in some cases) adults, continue to show a bias towards overconfidence (Roebers 2002; Roebers et al. 2007).

Children given pairs of objects to remember can be tested by presenting one object and asking whether they are likely to remember which object it was paired with. At a slightly younger age (around 6 years old), children are relatively accurate in these judgements of learning ('JoLs'), provided the question is asked after a delay (Schneider et al. 2000). ${ }^{5}$ However, children's errors are in the direction of overconfidence, especially when making predictions: preschool children are very overconfident when they predict how well they will recall items in the future (Lipko et al. 2009, 2012), but there is a marked change in overconfidence towards better calibration in the first years of school. At an older age (by 12 years old), children's JoLs are relatively accurate and do not change markedly thereafter (van Loon et al. 2013). Pre-school children are also very overconfident when making 'ease of learning' judgements, predicting in advance whether they will be able to remember a word list (Worden and Sladewski-Awig 1982; Yussen and Levy Jr 1975).

Overconfidence in younger children has also been detected in relation to concept learning. When learning new concepts, 8 years olds are overconfident in their JoLs regardless of their inability to generate sentences using the new concept, while older children (around 11 years old) show overconfidence when they can generate a test sentence for the new concept (van Loon et al. 2017). The presence of overconfidence in infants' monitoring of concept learning leads to less restudy time, which in turn has a detrimental impact on mastering new concepts (van Loon et al. 2017).

The two components of metacognition also show a developmental trend. Regarding control, there is a clear developmental trend in self-regulation - top-down control including planning, directing, and evaluating one's behaviour - and in the relation between monitoring and control (Schneider and Löffler 2016). Regarding monitoring and its use for control, the school-age child becomes better able to monitor their firstorder states and to make use of feelings of high versus low confidence to control their cognitive processes and guide their learning (Koriat and Ackerman 2010).

In summary, analytic metacognition grows more sophisticated throughout children's school years, with the explicit use of analogies, and many learning strategies, not appearing until 9-12 years old, so it plausibly plays a role only for later cases of conceptual change. Procedural metacognition appears much earlier and is largely stable by this age. Changes in procedural metacognition might affect the pattern of earlier cases of conceptual change. Many forms of procedural metacognition are characterised

\footnotetext{
${ }^{4}$ Although implicit measures might reveal conflict with explicit, verbal measures: infants who are good at monitoring their cognitive states may fail at reporting them in explicit measures (Kim et al. 2016).

${ }^{5}$ The benefit of delay in forming accurate JoLs is true of adults as well as children (Rhodes and Tauber 2011).
} 
by pronounced overconfidence in preschool children, which gives way to broadly accurate judgements of learning and confidence by 12 years old. In the next section we look at the possible role of metacognitive overconfidence in a process of conceptual change.

\section{Overconfidence and Metacognitive Disequilibration}

We saw in the previous section that children are initially highly overconfident and subsequently develop better calibration. A reduction in overconfidence is one metacognitive change that could cause an across the board change in the way concepts are acquired and revised. Overconfidence is tested by asking children to produce judgements of learning, that is predictions about how likely they will be to remember a learnt piece of information when given a cue.

When the child already has concepts in a domain they often become resistant to change. A period of disequilibration is needed before the child overcomes this resistance and acquires a new concept. This is true in many domains: naïve physics, intuitive biology, the astronomical concept of the earth, and the rational numbers. Disequilibration here indicates the detection of inconsistencies as a path to confidence reduction. As the study by van Loon et al. (2017) shows, overconfidence has a detrimental effect on the acquisition of new concepts. Could it also play a detrimental role in the revision of concepts?

The mere detection of a contradiction is not enough to drive a process of conceptual change: Carey (1999) describes the case of a 4-year-old child who notices a local contradiction in her concept of "living being" and yet fails to revise her concept. Why does a 4-year-old fail to use a contradiction to revise her concept? This might be due to overconfidence. It is thus important to distinguish between the mere detection of the presence of a contradiction (a process of 'local disequilibration' as used by Carey) and a cumulative process of metacognitive disequilibration. Metacognitive disequilibration is a matter of the child becoming aware that there are contradictions in their current beliefs and concepts, and that the predictions they make are not reliable.

Metacognitive disequilibration is not easy to achieve. Overconfidence stands in the way of becoming disequilibrated. ${ }^{6}$ Thus, the developmental change in procedural metacognition identified above, away from overconfidence and towards reasonably accurate metacognition by age 7 or 8 , is an essential precursor to the kinds of conceptual change that depend upon metacognitive disequilibration. ${ }^{7}$ For example, Karmiloff-Smith studied children's naïve physics, in the form of their understanding of the laws of torque involved in balancing bricks (Karmiloff-Smith 1991). By 6 or 7 years old, children had learnt a systematic rule for

\footnotetext{
${ }^{6}$ Overconfidence itself also calls for explanation. However, we don't here explore the origins of overconfidence. Are children overconfident because the old concept's use is more fluent (and thus easier to use), because they can't integrate knowledge (Kintsch 1994), or because of motivation? The relation between overconfidence and these factors is left for future research.

${ }^{7}$ Metacognitive disequilibration could come from a variety of sources: from the child's own errors, from others' instructions, and might also involve factors such as better executive functions or attentional control. These factors are important in development, but they do not seem to be the driving force behind conceptual change on their own: for example, Chinese children, who perform better on measures of executive functions than American children, still acquire theory of mind concepts at the same age (Sabbagh et al. 2006).
} 
solving this task, but they were overconfident about it, sticking with the rule even when it manifestly failed (when using asymmetrically weighted bricks). Only by 8 or 9 years old did children learn from experience about the rule's limitations. By this age overconfidence is reduced and children's confidence judgements about task performance have reached nearadult levels of accuracy.

At the stage when children are metacognitively unaware of contradictions they take on board contradictory claims and integrate them into a 'synthetic concept'. Children's initial conception of the earth is of a flat disk. By 8 years old they have learnt that the earth is a sphere that revolves around the sun, but they appear to integrate this belief into a synthetic model that also includes their initial beliefs. It is only later at 10 years old, when they become aware of the contradiction, that children acquire the scientific concept of the spherical earth (Vosniadou 1994; Vosniadou and Brewer 1992). For this transition, it seems that it occurs when the child is aware of the unreliability of their beliefs. Awareness of the unreliability of their beliefs seems thus needed to acquire the new concept. Metacognitive disequilibration is also a stepping stone towards acquiring the mature concept of a living thing by age 7 (Carey 1985; Crider 1981; Keil 1979), of blood circulation by 10 years old (Mason 1994) and of the rational numbers (between 8 and 12: Carey 2009, ch. 9).

Not all episodes of disequilibration lead to conceptual change. Sometimes the conflict is resolved by keeping incompatible explanations, but articulating them at different levels, or applying them to different aspects of the phenomenon (Legare and Shtulman 2018). Indeed, it's possible that once a new concept has been acquired, there is a period of competition between the new concept and the old one. The subsistence of co-existing explanations does not contradict the role of metacognition in conceptual change discussed in this paper: we only look at cases where inconsistency is resolved in a change and try to uncover the process that could explain why a child is stuck at that stage and what allows her to overcome it.

In short, a development in procedural metacognition in the early school years, away from overconfidence and towards better metacognitive accuracy, seems to have an across-the-board effect on the way concepts are acquired. The child has to learn new concepts in domains where they have concepts already. Their resistance is overcome when they become aware that their beliefs are contradictory and their predictions fail, a prerequisite for which is the loss of the strong bias towards overconfidence characteristic of procedural metacognition in pre-school children.

This is a hypothesis in need of testing. One possible test is to split children in a process of conceptual revision into two groups, and measure their confidence levels at the beginning, then intervene in one group to highlight inconsistencies between their old concept and new information, measure whether their confidence is reduced as a result, compare the process of conceptual revision in the two groups, and examine whether any differences in conceptual change are mediated by differences in confidence bias.

\section{Theory of Mind}

In the previous section we focused on a potential role of procedural metacognition for conceptual change. What about analytic metacognition? 
One way in which analytic metacognition could play a role in conceptual change is in the acquisition of a theory of mind. Theory of mind could be relevant to our question in one of two ways. First, acquisition of explicit theory of mind could depend on a prior change in metacognitive abilities. Second, self-application of theory of mind could itself be a metacognitive ability which has an across the board effect on subsequent conceptual development. We consider these in turn.

First off, is there some global change in metacognitive abilities that opens the way for the child to acquire a theory of mind, that is, explicit concepts of belief, desire, knowledge and intention? This question has not been systematically investigated (with the exception of Kim et al. 2018), but the brief review of changes in metacognitive abilities above does not throw up any obvious candidates. While there is considerable evidence (e.g. Onishi and Baillargeon 2005) that even infants are able to keep track of others' belief states in some ways (but cf. Heyes (2014)), children are not able to give accurate explicit reports of another person's false beliefs until between 3 and 4 years old (Ruffman and Perner 2005). One potential explanation is the development of executive function - not until this age have children developed sufficiently sophisticated executive capacities to deal with the demands of the task (Helming et al. 2016). The executive function development hypothesis for the failure to manifest an explicit mastery of mental states concepts has not received widespread support (Sabbagh et al. 2013). But even if there were a change in executive functions that had a global impact on conceptual development, it would not obviously be a change in metacognitive abilities.

Conversely, applying the concept of belief is a form of explicit metacognition, ${ }^{8}$ so we can ask whether its acquisition has a global effect on concept acquisition. Do children acquire new kinds of concept or learn concepts in new ways once they have an explicit theory of mind? Again, the prospects of a connection here are not promising. For example, understanding false mental representations does not appear to endow the child with a general purpose understanding that representations can be false in other domains. Acquisition of explicit theory of mind does not correlate with the age at which a child comes to understand that a photograph can be false, for example (Zaitchik 1990). ${ }^{9}$ 4-year-olds who were successful at the false belief task, performed at chance in the false photograph task.

Given the connection between being alive and having a mind, having a concept of belief seems like it could be a precursor to forming more sophisticated biological concepts, such as the concepts of agent and of living being, but there does not seem to be a tight connection. Children with Williams' syndrome pass the false belief task at around four years of age, thus showing standard development of a theory of mind and the possession of domain-specific metarepresentational abilities, but they do not show

\footnotetext{
${ }^{8}$ One such proposal for the relation between mindreading and explicit metacognition has been suggested by Perner, according to whom the acquisition of mental state concepts provides the child with metarepresentational abilities that she then uses in metacognition (Perner et al. 2015).

${ }^{9}$ In the experiment, an actor places an object in location A and takes a photo of it; then another actor moves the object to location B, and the test question to the child is: "In the picture, where is the object?". This experimental design mirrors the one used to test understanding of false beliefs. Perner and Leekam (2008) argue that the false photograph test in reality does not test children's understanding of "falsity", because, according to them, the photograph is in fact a true representation of the situation at the time it was taken in the past. Zaitchik, on the other hand, argues that the false photograph test detects a difficulty in understanding representations.
} 
the same path to conceptual change in the domain of intuitive biology. They do not change their concept of a living being between 6 and 10 years of age (which is roughly: an object capable of self-propelled motion). They apply it to cars, while children in the same age range who don't have Williams' syndrome correctly categorize cars as being non-living (Carey and Johnson 2000). According to Carey and Johnson this shows that theory of mind does not "make available the machinery to support conceptual change" (p. 231) and that theory of mind is not a precondition for conceptual change in naïve biology.

Both these cases suggest that acquiring theory of mind is a matter of getting some domain-specific concepts (believe, know, desire, intend) and thus an understanding of metarepresentation in a domain-specific way, as well as acquiring a set of beliefs about how the mind works, without producing a deeper underlying change in the ability to acquire concepts in general.

This does not mean that there is no connection at all between the acquisition of a theory of mind and metacognition. Indeed, there is an impact of theory of mind on analytic metacognition (Lockl and Schneider 2007), but that is unsurprising since analytic metacognition partly involves making use of the concept of belief (and other mental state concepts). To the extent that sophisticated analytic metacognition opens up new ways of learning concepts - like the explicit use of analogies in learning new scientific concepts - acquiring explicit theory of mind is an early staging post on the way to this much later outcome, but it is not the only ingredient in the way metacognition is involved in these later conceptual changes. We examine these in the next section. They draw on richer forms of analytic metacognition, that may or may not involve mindreading.

Apart from domain-specific impacts of the mastery of mental state terms on analytic metacognition, does theory of mind influence procedural metacognition as well? This question hasn't been investigated in-depth, but recent evidence suggests a negative answer: success in false belief tasks does not seem related to success in tasks involving procedural metacognition, for meta-perception (Bernard, Proust \& Clément 2015) and for meta-memory (Balcomb and Gerken 2008).

\section{Analogising}

Another way in which analytic metacognition could play a role in conceptual change is via the use of analogies. This role has been extensively studied in the educational literature. Using and recognising analogies is crucial to the way children learn many scientific and mathematical concepts. It is one of the most studied tools for teaching and learning in the educational literature. The use of analogies in learning has (with some exceptions) a positive influence on learning (Vosniadou and Skopeliti 2019). An analogy involves two concepts (usually from different domains) and a mapping between the two, but without a combination or integration of the two concepts. Using an analogy might not require metacognition, but the reflective use of analogies in learning is usually explicitly taught in an educational setting and makes use of metacognitive strategies, such as understanding how one conceptualises a domain, and grasping the educational goal of the analogy in explaining a phenomenon. 
Analogy alone is not sufficient for driving conceptual change: other factors are involved, such as the student's prior knowledge (Vosniadou \& Skopeliti, forthcoming), but, in conjunction with other factors, analogies play a role in structuring the learning environment and in conceptual change. Mason (1994) describes the way children use an analogy with the postal system, applied metacognitively, to learn a sophisticated conception of blood circulation at 10 years old. What they acquire is a concept of blood circulation that is interrelated with the heart and blood vessels. Acquiring concepts of the rational numbers, typically between 8 and 12 years old, also draws on the child's capacity to understand and use analogies (Carey 2009, ch. 9). ${ }^{10}$ Analogy use seem to be more effective in changing concepts in cases when children (and adults) not only use the analogy to acquire knowledge about a new domain, but are also aware of the nature and instructional purpose of the analogy for learning, of the goal of the analogy with respect to the specific domain in question, and of their initial and final conceptions. These strategies require the use of analytic metacognition (Mason 1994; Nersessian 2010).

Most studies have focused on the use of analogies in teaching by the instructor (Duit 1991; Glynn et al. 2012; Harrison and Treagust 2006) or in adults (college age students and experts) (Clement 1987, 1991, 2013). In this context we can see how using analogies for introducing new concepts makes use of metacognition. For example, Glynn et al. (2012) suggest a teaching method for introducing the concept of pulsar by analogising it to the rotating lamp in a lighthouse. The teacher introduces the target concept, the analogue concept and its relevant features, a mapping of similarities, but he also discusses the goal of the analogy and its limitations in understanding the target concept. Learning new concepts through analogies is especially fruitful for scientific novices and helps the learner acquire knowledge that can be used to answer inferential questions about the domain and not just basic-knowledge questions, showing that the concept can now be used for inferential reasoning (Donnelly and McDaniel 1993).

One worry with thinking of explicit analogy use as calling for analytic metacognition is that educational studies involve a teacher's explicit instructions in how to use and apply an analogy. In our view the application and use of an analogy can often involve analytic metacognition, whether that analogy is produced spontaneously or taught explicitly. Spontaneous production of analogies may indeed engage metacognition in interesting ways, and although we are not aware of studies of spontaneous use of analogies in children, spontaneous analogy use and its impact on conceptual change has been explored in college-age students (Clement 1987, 2013). Spontaneous analogy production can also sometimes be detrimental to conceptual change, if the analogy reinforces the starting conceptual point rather than helping the thinker to overcome it (Clement 2013). Analytic metacognition might help the learner to guide the way the use analogies so as to avoid this pitfall (if all goes well).

Recognising a similarity between domains need not be a metacognitive operation. For example, children spontaneously make the analogical inference from plants being goal-directed (like animals) to plants also being living beings, without explicit

\footnotetext{
${ }^{10}$ Carey also argues that analogising is at work when children acquire concepts of natural numbers (Carey 2009, ch. 8). This happens much earlier, around 3 years old. However, this does not appear to involve the ability to understand explicit analogies. It may instead draw on a more implicit process by which a mapping is established between sets of symbols.
} 
comparison or feedback (Opfer and Siegler 2004). However, understanding an analogy as a way of thinking about a domain does call for metacognition. Where children draw on analogies to acquire new scientific and mathematical concepts, from 8 or 9 years old onwards, they appear to be using explicit metacognitive considerations to understand the nature and instructional purpose of the analogy. They are also plausibly thinking explicitly about the way they themselves conceptualise the domain and can compare their previous conceptualisation with the new one. Thus, this is a case where an increase in the sophistication of children's analytic metacognitive abilities has an across-the-board effect on the way they acquire new concepts.

\section{Predictions}

We have suggested two examples where a change in metacognitive ability opens up a new stage in the way the child can acquire new concepts: one earlier, in procedural metacognition, and one later, in analytic metacognition. ${ }^{11}$ Various predictions follow from these hypotheses.

Most obviously, in line with current educational research (Harrison and Treagust 2006; Mason 1994; Vosniadou and Skopeliti 2019), we predict that individual differences in children's facility in using explicit analogies, typically in the later years of elementary school, will trigger differences in how well the individuals acquire sophisticated scientific and mathematical concepts. For instance, in their experiment on the influence on analogies on understanding, Vosniadou and Skopeliti compare two groups: one group read a scientific text without the analogy, while the other group read a text with an analogy. In the latter group differences were found in respect to the understanding of the analogy (from no understanding to partial understanding to complete understanding): one way of testing the prediction would be to compare incremental conceptual change among groups split by how well they understand the analogy.

More of a surprise is that there should be a connection between improvements in procedural metacognition and a very specific way of acquiring new concepts, i.e. in the process by which children get a new concept when they already have a somewhat adequate conceptual understanding of a domain. Our hypothesis predicts that individual differences in calibration - reduced overconfidence in confidence judgements and JoLs - should correlate with the age at which the child begins to acquire new concepts through metacognitive disequilibration.

Both of these changes take place while children are in formal education and have overwhelmingly been studied in Europe and America. Looking at these processes cross-culturally would tell us a lot more about why they come on stream and how culture influences them (Proust and Fortier 2018). For example, in some tasks Mayan children differ in confidence levels from German and Japanese children (Kim et al. 2018). There are also cultural differences in the development of analytic metacognition:

\footnotetext{
${ }^{11}$ Important questions for future research also arise for the potential role of metacognition in concept use, which we set aside in the introduction; also whether metacognition plays different roles as between concept acquisition, concept revision and concept replacement.
} 
German children manifest meta-memory regarding strategies at an earlier age than American children (Schneider and Löffler 2016). If the changes in analytic and procedural metacognition we have pointed to occur at different ages in different cultures, then cross-cultural comparisons at the group level are another way to test the correlations predicted in the last paragraph.

More generally, we predict that variations in confidence calibration (connected with different factors, such as age or culture, among others) will have an impact on conceptual change. As we have seen, overconfidence can act as an obstacle to conceptual change. What is needed for a better calibration in a process of conceptual change? Is minimal task experience enough (Lipowski et al. 2013)? Or is the calibration process a long developmental trend? There are ways of lowering confidence by repetition which are effective in adults, as shown by the underconfident-with-practice effect (Koriat et al. 2002), an effect that children do not show (Lipko et al. 2012). Yet, there is a developmental trend of improved calibration with respect to accuracy that continues beyond childhood: for example older adults do not show the underconfidentwith-practice effect, but are rather accurate in their JoLs, without showing overconfidence like children (Rast and Zimprich 2009). While confidence calibration has been studied in connection to learning it would be interesting to explore how it impacts conceptual change and how differences in metacognitive accuracy might explain differences in the rates of conceptual change.

\section{Conclusion}

We have identified possible connections between stages in metacognitive development and conceptual change in children. We focused on the question whether developmental changes in metacognition have an across the board impact on conceptual development. There is a recognised connection between analytic metacognition and conceptual change: in the case we examined, children who are better at using explicit analogies in acquiring new concepts seem to change their concepts more easily. Our novel suggestion is that procedural metacognition also has a role in conceptual change. We reviewed evidence suggesting that changes in procedural metacognition, away from generalised overconfidence and towards better calibration, are important in allowing the child to become disequilibrated and thus open to new concepts in domains where they already possess functioning concepts. This example suggests there may well be other ways in which children's metacognitive development produces wholescale changes in the way they are able to acquire new concepts. There has been little systematic investigation of these potentially interesting links, especially in the emerging domain of procedural metacognition in childhood. As we have shown, there is good reason to expect that this investigation will prove to be fruitful.

Acknowledgements We are thankful to two anonymous reviewers from The Review of Philosophy and Psychology for their helpful comments and to Joëlle Proust for extensive feedback on a previous draft of this paper. This project has received funding from the European Research Council (ERC) under the European Union's Horizon 2020 research and innovation programme grant Agreement No. 681422. 
Open Access This article is licensed under a Creative Commons Attribution 4.0 International License, which permits use, sharing, adaptation, distribution and reproduction in any medium or format, as long as you give appropriate credit to the original author(s) and the source, provide a link to the Creative Commons licence, and indicate if changes were made. The images or other third party material in this article are included in the article's Creative Commons licence, unless indicated otherwise in a credit line to the material. If material is not included in the article's Creative Commons licence and your intended use is not permitted by statutory regulation or exceeds the permitted use, you will need to obtain permission directly from the copyright holder. To view a copy of this licence, visit http://creativecommons.org/licenses/by/4.0/.

\section{References}

Aslin, R.N. 2007. What's in a look? Developmental Science 10 (1): 48-53.

Balcomb, F.K., and L. Gerken. 2008. Three-year-old children can access their own memory to guide responses on a visual matching task. Developmental Science 11 (5): 750-760.

Beran, M., J. Brandl, J. Perner, and J. Proust. 2012. The Foundations Of Metacognition. Oxford University Press.

Blank, L.M. 2000. A metacognitive learning cycle: A better warranty for student understanding? Science Education 84 (4): 486-506. https://doi.org/10.1002/1098-237x(200007)84:4<486::Aid-sce4>3.0.Co;2-u.

Carey, S. 1985. Conceptual change in childhood. Cambridge: MIT Press.

Carey, S. 1999. Sources of conceptual change. In Conceptual development: Piaget's legacy, eds. E.K. Scholnik, K. Nelson, S.A. Gelman and P.H. Miller, 293-326. Psychology Press.

Carey, S. 2000. Science education as conceptual change. Journal of Applied Developmental Psychology 21 (1): 13-19.

Carey, S. 2009. The origin of concepts. Oxford: Oxford University Press.

Carey, S., and S. Johnson. 2000. Meta-representation and conceptual change: Evidence from Williams syndrome. In Meta-representations: A multidisciplinary perspective, ed. D. Sperber, 225-264. New York: Oxford University Press.

Carey, S., and E. Spelke. 1994. Domain-specific knowledge and conceptual change. In Mapping the mind: Domain specificity in cognition and culture, ed. L.A. Hirschfeld and S.A. Gelman, 169-200. Cambridge: Cambridge University Press.

Chen, S., and H. Schildberg-Hörisch. 2019. Looking at the bright side: The motivation value of overconfidence. European Economic Review 120.

Clement, J. 1987. Generation of spontaneous analogies by students solving science problems. In Thinking across cultures, ed. D.M. Topping, D.C. Crowell, and V.N. Kobayashi, 303-308. New York and London: Routledge.

Clement, J. 1991. Nonformal reasoning in experts and in science students: The use of analogies, extreme cases, and physical intuition. In Informal reasoning and education, ed. J.F. Voss, D.N. Perkins, and J.W. Segal, vol. 1, 345-362. New York and London: Routledge.

Clement, J. 2013. Roles for explanatory models and analogies in conceptual change. In International handbook of research on conceptual change, ed. S. Vosniadou, 412-446. New York and London: Routledge.

Crider, C. 1981. Children's conceptions of the body interior. In New directions for child and adolescent development, ed. R. Bibace and M. Walsh, 49-65. San Francisco: Jossey-Bass.

Donnelly, C.M., and M.A. McDaniel. 1993. Use of analogy in learning scientific concepts. Journal of Experimental Psychology: Learning, Memory, and Cognition 19 (4): 975.

Duit, R. 1991. On the role of analogies and metaphors in learning science. Science Education 75 (6): 649-672.

Flavell, J. 1979. Metacognition and cognitive monitoring: A new area of cognitive-developmental inquiry. American Psychologist 34 (10): 906-911. https://doi.org/10.1037/0003-066X.34.10.906.

Flavell, J., \& Wellman, H. (1975). Metamemory. Paper presented at the Annual Meeting of the American Psychological Association Chicago, Ill.

Geurten, M., and C. Bastin. 2019. Behaviors speak louder than explicit reports: Implicit metacognition in 2.5year-old children. Developmental Science 22 (2): e12742.

Glynn, S.M., R. Duit, and R.B. Thiele. 2012. Teaching science with analogies: A strategy for constructing knowledge. In Learning science in the schools, ed. S.M. Glynn and R. Duit, 259-286. Routledge.

Goupil, L., and S. Kouider. 2016. Behavioral and neural indices of metacognitive sensitivity in preverbal infants. Current Biology 26 (22): 3038-3045.

Goupil, L., M. Romand-Monnier, and S. Kouider. 2016. Infants ask for help when they know they don't know. Proceedings of the National Academy of Sciences 113 (13): 3492-3496. 
Hampton, R.R. 2009. Multiple demonstrations of metacognition in nonhumans: Converging evidence or multiple mechanisms? Comparative Cognition \& Behavior Reviews 4: 17-28.

Harris, P.L., D.T. Bartz, and M.L. Rowe. 2017. Young children communicate their ignorance and ask questions. Proceedings of the National Academy of Sciences 114 (30): 7884-7891.

Harrison, A.G., and D.F. Treagust. 2006. Teaching and learning with analogies. In Metaphor and analogy in science education, ed. P.J. Aubusson, A.G. Harrison, and S.M. Ritchie, 11-24. Dordrecht: Springer Netherlands.

Haukås, Å., C. Bjørke, and M. Dypedahl. 2018. Metacognition in language learning and teaching. Routledge.

Helming, K.A., B. Strickland, and P. Jacob. 2016. Solving the puzzle about early belief-ascription. Mind and Language 31 (4): 438-469.

Hennessey, G., Beeth, M., \& Zietsman, A. (1996). Teaching from a constructivist paradigm: A way of knowing and learning or a case of "pedagogical tricks?". Paper presented at the Annual Meeting of the National Association for Research in Science Teaching, St Louis, MO.

Heyes, C. 2014. False belief in infancy: A fresh look. Developmental Science 17 (5): 647-659. https://doi. org/10.1111/desc.12148.

Karmiloff-Smith, A. 1991. Beyond modularity: Innate constraints and developmental change. In The epigenesis of mind: Essays on biology and cognition, ed. S. Carey and R. Gelman, 171-197. New York and London: Psychology Press.

Keil, F. 1979. Conceptual Development. Harvard University Press.

Keil, F. 1989. Concepts, kinds, and cognitive development. Cambridge: The MIT Press.

Kim, S., M. Paulus, B. Sodian, and J. Proust. 2016. Young children's sensitivity to their own ignorance in informing others. PLoS One 11 (3): e0152595.

Kim, S., A. Shahaeian, and J. Proust. 2018. Developmental diversity in mindreading and metacognition. In Metacognitive diversity: An interdisciplinary approach, ed. J. Proust and M. Fortier, 97-133. Oxford University Press.

Kintsch, W. 1994. Text comprehension, memory, and learning. American Psychologist 49 (4): 294-303.

Koriat, A. 2016. Metacognition: Decision-making processes in self-monitoring and self-regulation. In The Wiley Blackwell handbook of judgment and decision making, ed. G. Keren and G. Wu, vol. 1, 356-379. Malden: Wiley-Blackwell.

Koriat, A., and R. Ackerman. 2010. Choice latency as a cue for children's subjective confidence in the correctness of their answers. Developmental Science 13 (3): 441-453.

Koriat, A., L. Sheffer, and H. Ma'ayan. 2002. Comparing objective and subjective learning curves: Judgments of learning exhibit increased underconfidence with practice. Journal of Experimental Psychology: General 131 (2): 147-162.

Krebs, S.S., and C.M. Roebers. 2012. The impact of retrieval processes, age, general achievement level, and test scoring scheme for children's metacognitive monitoring and controlling. Metacognition and Learning 7 (2): 75-90.

Kreutzer, M.A., C. Leonard, J.H. Flavell, and J.W. Hagen. 1975. An interview study of children's knowledge about memory. Monographs of the Society for Research in Child Development 40 (1): 1-60.

Legare, C.H., and A. Shtulman. 2018. Explanatory pluralism across cultures and development. In Metacognitive Diversity: An Interdisciplinary Approach, ed. J. Proust and M. Fortier, 415-432. Oxford University Press.

Lipko, A.R., J. Dunlosky, and W.E. Merriman. 2009. Persistent overconfidence despite practice: The role of task experience in preschoolers' recall predictions. Journal of Experimental Child Psychology 103 (2): $152-166$.

Lipko, A.R., J. Dunlosky, S.L. Lipowski, and W.E. Merriman. 2012. Young children are not underconfident with practice: The benefit of ignoring a fallible memory heuristic. Journal of Cognition and Development 13 (2): 174-188.

Lipowski, S.L., W.E. Merriman, and J. Dunlosky. 2013. Preschoolers can make highly accurate judgments of learning. Developmental Psychology 49 (8): 1505-1516.

Lockl, K., and W. Schneider. 2007. Knowledge about the mind: Links between theory of mind and later Metamemory. Child Development 78 (1): 148-167.

Lyons, K.E., and S. Ghetti. 2011. The development of uncertainty monitoring in early childhood. Child Development 82 (6): 1778-1787.

Mason, L. 1994. Cognitive and metacognitive aspects in conceptual change by analogy. Instructional Science 22 (3): 157-187.

Moynahan, E.D. 1978. Assessment and selection of paired associate strategies: A developmental study. Journal of Experimental Child Psychology 26 (2): 257-266.

Nersessian, N.J. 2010. Creating scientific concepts. Cambridge: MIT Press. 
Onishi, K.H., and R. Baillargeon. 2005. Do 15-month-old infants understand false beliefs? Science 308 (5719): 255-258. https://doi.org/10.1126/science.1107621.

Opfer, J.E., and R.S. Siegler. 2004. Revisiting preschoolers' living things concept: A microgenetic analysis of conceptual change in basic biology. Cognitive Psychology 49 (4): 301-332.

Özsoy, S. 2017. Is the earth flat or round? Primary school children's understandings of the planet earth: The case of Turkish children. International Electronic Journal of Elementary Education 4 (2): 407-415.

Perner, J., and S. Leekam. 2008. The curious incident of the photo that was accused of being false: Issues of domain specificity in development, autism, and brain imaging. The Quarterly Journal of Experimental Psychology 61 (1): 76-89.

Perner, J., M. Huemer, and B. Leahy. 2015. Mental files and belief: A cognitive theory of how children represent belief and its intensionality. Cognition 145: 77-88.

Proust, J. 2010. Metacognition. Philosophy Compass 5 (11): 989-998.

Proust, J. 2013. The philosophy of metacognition: Mental agency and self- awareness. Oxford: Oxford University Press.

Proust, J., and M. Fortier. 2018. Metacognitive Diversity: An Interdisciplinary Approach. Oxford University Press.

Rast, P., and D. Zimprich. 2009. Age differences in the Underconfidence-with-practice effect. Experimental Aging Research 35 (4): 400-431. https://doi.org/10.1080/03610730903175782.

Rhodes, M.G., and S.K. Tauber. 2011. The influence of delaying judgments of learning on metacognitive accuracy: A meta-analytic review. Psychological Bulletin 137 (1): 131-148.

Roebers, C.M. 2002. Confidence judgments in children's and adult's event recall and suggestibility. Developmental Psychology 38 (6): 1052-1067.

Roebers, C.M., N. von der Linden, W. Schneider, and P. Howie. 2007. Children's metamemorial judgments in an event recall task. Journal of Experimental Child Psychology 97 (2): 117-137.

Ruffman, T., and J. Perner. 2005. Do infants really understand false belief? Trends in Cognitive Sciences 9 (10): 462-463.

Sabbagh, M., F. Xu, S.M. Carlson, L.J. Moses, and K. Lee. 2006. The development of executive functioning and theory of mind: A comparison of Chinese and US preschoolers. Psychological Science 17 (1): 74-81.

Sabbagh, M., J. Benson, and V. Kuhlmeier. 2013. False-belief understanding in infants and preschoolers. In The Infant Mind: Origins of the Social Brain, ed. M. Legerstee, D. Haley, and M. Bornstein. Guilford Press.

Schneider, W. 1998. Performance prediction in young children: Effects of skill, metacognition and wishful thinking. Developmental Science 1 (2): 291-297.

Schneider, W., and E. Löffler. 2016. The development of metacognitive knowledge in children and adolescents. In The Oxford handbook of metamemory, ed. J. Dunlosky and S.K. Tauber, 491-518. New York: Oxford University Press.

Schneider, W., and M. Pressley. 2013. Memory development between two and twenty. Psychology Press.

Schneider, W., M. Visé, K. Lockl, and T.O. Nelson. 2000. Developmental trends in children's memory monitoring: Evidence from a judgment-of-learning task. Cognitive Development 15 (2): 115-134.

Schwarz, N. 2012. Feelings-as-information theory. In Handbook of theories of social psychology, ed. P.A.M. van Lange, A.W. Kruglanski, and E.T. Higgins, vol. 1, 289-308. SAGE.

Shea, N. 2019. Concept-metacognition. Mind and Language.

Sinatra, G.M., and P.R. Pintrich. 2003. The role of intentions in conceptual change learning. In Intentional Conceptual Change, ed. G.M. Sinatra and P.R. Pintrich, 1-18. L. Erlbaum.

Smith, C., J. Snir, and L. Grosslight. 1992. Using conceptual models to facilitate conceptual change: The case of weight-density differentiation. Cognition and Instruction 9 (3): 221-283.

Sodian, B., and W. Schneider. 1999. Memory strategy development-gradual increase, sudden insight, or roller coaster? In Individual development from 3 to 12: Findings from the Munich Longitudinal Study, ed. F.E. Weinert and W. Schneider, 61-77. Cambridge University Press.

Son, L. 2004. Spacing one's study: Evidence for a metacognitive control strategy. Journal of Experimental Psychology: Learning, Memory, and Cognition 30 (3): 601-604. https://doi.org/10.1037/0278-7393.30.3.601.

Spelke, E.S., and G.A. Van de Walle. 1993. Perceiving and reasoning about objects: Insights from infants. In Spatial representation: Problems in philosophy and psychology, ed. N. Eilan, R. McCarthy, and B. Brewer, 132-161. Cambridge: Blackwell Publishing.

Strevens, M. 2019. Thinking off your feet : how empirical psychology vindicates armchair philosophy, 2019. Cambridge: The Belknap Press of Harvard University Press.

Thagard, P. 1992. Analogy, explanation, and education. Journal of Research in Science Teaching 29 (6): $537-$ 544.

Thagard, P. 2018. Conceptual revolutions. Princeton University Press. 
Thomas, G.P. 2012. Metacognition in science education: Past, present and future considerations. In Second international handbook of science education, ed. B.J. Fraser, K. Tobin, and C.J. McRobbie, 131-144. Dordrecht: Springer Netherlands.

Van Kraayenoord, C. 2010. The role of metacognition in reading comprehension. In Focal points of the research and development of pedagogically-psychological perspectives, ed. H. Trolldenier, W. Lenhard, and P. Marx, 277-302. Gottingen: Hogrefe.

van Loon, M., A. de Bruin, T. van Gog, and J. van Merriënboer. 2013. Activation of inaccurate prior knowledge affects primary-school students' metacognitive judgments and calibration. Learning and Instruction 24: 15-25.

van Loon, M., A. de Bruin, J. Leppink, and C. Roebers. 2017. Why are children overconfident? Developmental differences in the implementation of accessibility cues when judging concept learning. Journal of Experimental Child Psychology 158: 77-94.

Vicente, A., and F. Martínez Manrique. 2014. The big concepts paper: A defence of hybridism. The British Journal for the Philosophy of Science 67 (1): 59-88.

Von der Linden, N., and C.M. Roebers. 2006. Developmental changes in uncertainty monitoring during an event recall task. Metacognition and Learning 1 (3): 213-228.

Vosniadou, S. 1994. Universal and culture-specific properties of children's mental models of the earth. In Mapping the mind: Domain specificity in cognition and culture, ed. L.A. Hirschfeld and S.A. Gelman, 412-430. Cambridge: Cambridge University Press.

Vosniadou, S. 2007. Conceptual change and education. Human Development 50 (1): 47-54. https://doi. org/10.1159/000097684.

Vosniadou, S., and W.F. Brewer. 1992. Mental models of the earth: A study of conceptual change in childhood. Cognitive Psychology 24 (4): 535-585.

Vosniadou, S., and I. Skopeliti. 2019. Evaluating the effects of analogy enriched text on the learning of science: The importance of learning indexes. Journal of Research in Science Teaching 56 (6): 732-764. https://doi.org/10.1002/tea.21523.

Weiskopf, D.A. 2009. The plurality of concepts. Synthese 169 (1): 145.

Wellman, H.M., and S.A. Gelman. 1998. Knowledge acquisition in foundational domains. In Handbook of child psychology: Cognition, perception, and language, ed. W. Damon, vol. 2, 523-573. Wiley.

Worden, P.E., and L.J. Sladewski-Awig. 1982. Children's awareness of memorability. Journal of Educational Psychology 74 (3): 341.

Xu, F., and J.B. Tenenbaum. 2007. Word learning as Bayesian inference. Psychological Review 114 (2): $245-272$.

Yussen, S.R., and V.M. Levy Jr. 1975. Developmental changes in predicting one's own span of short-term memory. Journal of Experimental Child Psychology 19 (3): 502-508.

Zaitchik, D. 1990. When representations conflict with reality: The preschooler's problem with false beliefs and “false" photographs. Cognition 35 (1): 41-68. https://doi.org/10.1016/0010-0277(90)90036-J.

Publisher's Note Springer Nature remains neutral with regard to jurisdictional claims in published maps and institutional affiliations. 